\title{
Experimental Study of a New Technique to Reduce Absorber Heat Losses: Applied for Drying Painted Gas Cylinders
}

\author{
${ }^{1}$ Driss Slimani, ${ }^{1}$ Bachir Elkihel and ${ }^{2}$ Christelle Perilhon \\ ${ }^{1}$ Department of Industrial Engineering, Engineering School of Applied Sciences, \\ Mohamed First University, Oujda, Morocco \\ ${ }^{2}$ Laboratory Molecular Chemistry, Chemical Process Engineering and Energetics, \\ National Conservatory of Arts and Crafts, 75141 Paris Cedex 03, France
}

\begin{abstract}
In this experimental study, a solar dryer has been designed and manufactured by combining a concave solar concentrator with a series of convergent lenses whose concentrated radiation are reflected to the same absorber. Our main goal for this technique is to reduce the absorber thermal losses without the need to provide expensive and fragile thermal insulation. Thus, the idea is to create the greenhouse heating on both sides of the flat absorber. In our dryer, the receiver uses air and water as the heat transfer fluid these two fluids are heated in the absorber and sent a heat exchanger. Furthermore, in order to improve the thermal performance, our approach is based on some techniques such as the combination of two types of solar concentrators, simultaneous use of two natural heat transfer fluids, tilting concentrators, the insertion of obstacles at the absorber and a specific solar tracker for explosive atmosphere. Our dryer has been tested for the first time in gas filling plant in Morocco for drying painted gas bottles. Compared with flat-plate solar collector, the proposed dryer has improved the drying temperature from $40-85^{\circ} \mathrm{C}$. In fact, our solar dryer has reduced the drying time from 420 to just $40 \mathrm{sec}$. Also, the health risks of workers have been reduced and the number of painted bottles has been increased by more than $43 \%$.
\end{abstract}

Key words: Combined solar concentrators, solar dryer, two naturel heat transfer fluids, thermal losses, obstacles, tilting concentrators

\section{INTRODUCTION}

The most important property of the solar energy is a renewable energy resource. The average annual sun exposure in Morocco is about $2500 \mathrm{~h}$ it receives a daily solar energy of 16.2-27 MJ/m² (Bennamoun and Belhamri, 2007). The drying consumes about $12-25 \%$ of the total primary energy demand (Keey, 1992). The solar energy is an area to study extremely in drying due to they are easily applicable. Therefore, this energy appears to be the energy of the future (Teyeb et al., 2006). The solar collector constitutes the major component of any solar system (Bader et al., 2011). Hot air is obtained from these collectors and they are used in space heating (Christensen, 1998), product drying (Karim and Hawlader, 2004; Fudholi et al., 2011), greenhouse heating (Benli and Durmus, 2009) and pre-heating in ventilation systems (Sokhansanj and Schoenau, 1991; Cordeau, 2010). The parabolic solar concentrator is often used with systems for solar absorption refrigeration that can achieve temperatures over $100^{\circ} \mathrm{C}$ (Chekirou et al.,
2007; Gama et al., 2008). Thus, the environmental problems related to the emission of greenhouse gases will be reduced (Blair et al., 2005).

In this context, the solar drying systems must be properly designed to meet the drying requirements such as product quality and drying time. Several researchers have developed simulation models for natural convection and forced convection systems (Youcef and Desmons, 2006). Solar air collectors are simple devices for air heating by utilizing solar energy for many applications that require low to moderate temperatures below $60^{\circ} \mathrm{C}$. The principal types were classified as one pass, double duct and two passes (Forson et al., 2003). Recently, studies covering different types of collectors have been undertaken by several researchers. Hot air generation and drying applications were examined with their designs (Turgut and Onur, 2000; Momin et al., 2002). A single-pass solar air collector (Bulut and Durmaz, 2006) and a double-pass solar air collector were designed and experimentally analyzed for their performance (Fudholi et al., 2011; Ho et al., 2011).

Corresponding Author: Driss Slimani, Department of Industrial Engineering, Engineering School of Applied Sciences, Mohamed First University, Oujda, Morocco 
The activity of painting gas bottles is expanding in Morocco. Despite, this evolution, drying is done naturally and at ambient temperature. However, this traditional drying generates two major drawbacks. First, the customer expectations in terms of time, quality and quantity are not met. Second, in terms of employee health, exposure to high concentrations of solvents can cause loss of medium-term and long-term knowledge, affecting mood and memory (Beach et al., 1997; Pronk et al., 2007). To overcome these drawbacks in this research and for the first time in this industry, we propose a solar dryer by combining a concave solar concentrator with a series of convergent lenses whose concentrated radiation are reflected to the same absorber.

In this study, we present the design and experimental studies of a new dryer including the heating unit combines a parabolic trough concentrator with a series of convergent lenses for the same absorber using a specific solar tracker for explosive atmosphere. Our main goalis to create the greenhouse heating effect on the lower and upper side of the planar absorber. Thus, the additional thermal energy provided by the convergent lenses makes it possible to compensate for thermal losses without using an expensive and fragile thermal insulation. The receiver design uses air and water as the heat transfer fluid these two fluids are heated and sent a heat exchanger to raise the drying air temperature. Also, to improve thermal performance and achieve air temperature that can ensure rapid drying, we used three techniques. Firstly, the combination of a parabolic trough concentrator with a series of convergent lenses for the same absorber. The simultaneous use of water and air as heat transfer fluid, so, these two fluids are heated in the absorber and sent a heat exchangerto raise the drying air temperature. Secondly, the insertion of the obstacles inside the absorber in addition while tilting the concentrators according to the latitude angle. Finally, the solar tracker realizedis specific to the explosive environment by using a pneumatic motor and a pneumatic control. Several experiments were conducted on a prototype designed and fabricated in a gas filling plant in Oujda (Morocco). The experimental results are compared to the flat absorber dryer and dryer to those whose effect will was created on a single side of the absorber.

\section{MATERIALS AND METHODS}

System design: In this study, we used a solar dryer equipped with acombined parabolic solar concentrator and a series of convergent lenses, forced convection and specific solar trackingfor an explosive atmosphere zone. Maximum power into the solar collector occurs when the

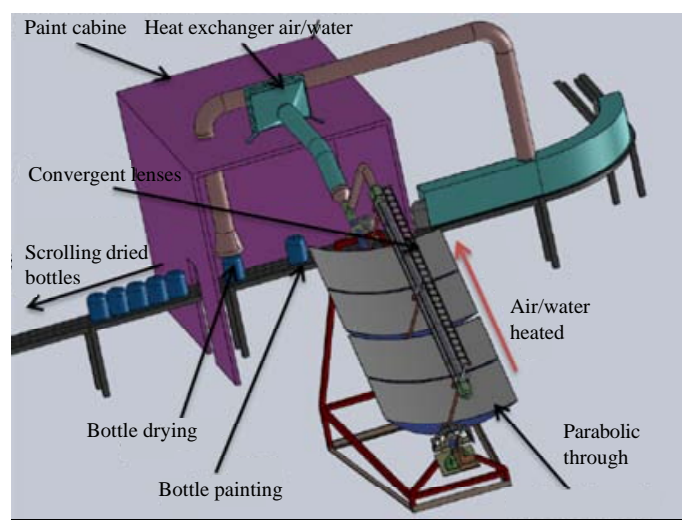

Fig. 1: Schematic view of the solar dryer used for painted gas cylinders

Table 1: The concentrator's characteristics

\begin{tabular}{ll}
\hline Components/Dimensions & Values \\
Concentrator & \\
Length, L & $3(\mathrm{~m})$ \\
Width, Lar & $1.8(\mathrm{~m})$ \\
Opening area & $5.1\left(\mathrm{~m}^{2}\right)$ \\
Absorber & \\
Thickness of the glass (e) & $0.005(\mathrm{~m})$ \\
Height obstacles (has $)$ & $0.04(\mathrm{~m})$ \\
Distance between obstacles (b) & $0.1(\mathrm{~m})$ \\
Absorber tube diameter (d) & $0.012(\mathrm{~m})$ \\
Absorber tube length (La) & $9(\mathrm{~m})$ \\
Convergent lenses & \\
Diameter & $90(\mathrm{~mm})$ \\
Number & 27 \\
\hline
\end{tabular}

surface is normal to the incident solar radiation. However, it is not always possible with fixed solar collector, since, the relative position of the Earth to the Sun varies (Honsberg, 2008). This system as shown in Fig. 1-3 was designed, fabricated and tested in a gas filling plant in Morocco. Theparabolic trough concentrator having an area $6 \mathrm{~m}^{2}(3 \times 2 \mathrm{~m})$ combined with 27 convergent lensesfor heatingair and waterat the same time. The receiver is connected to a drying unit through a centrifugal fan with an airflow rate up to $2000 \mathrm{~m}^{3} / \mathrm{h}$ (Fig. 4, Table 1). The absorber tube was fixed on galvanized sheet metal obstacles were inserted throughout the absorber tube for improved air heat exchange. The absorber was coated with black paint to absorb the incident solar radiation. The drying system used was the subject of some improvements in particular, thermal energy is generated simultaneously by the greenhouse on both sides of the absorber by the two concentrators. This technique compensates heat lost and allows the insertion of obstacles on both sides of the planar absorber as shown in Fig. 5.

The air is preheated at the absorber, afterits temperature will be increased at the exchanger (steam/air) at the exit of the receiver. The concave solar concentrator was tilted to an angle about $34^{\circ}$ horizontally which is considered an optimum angle for year-round performance 


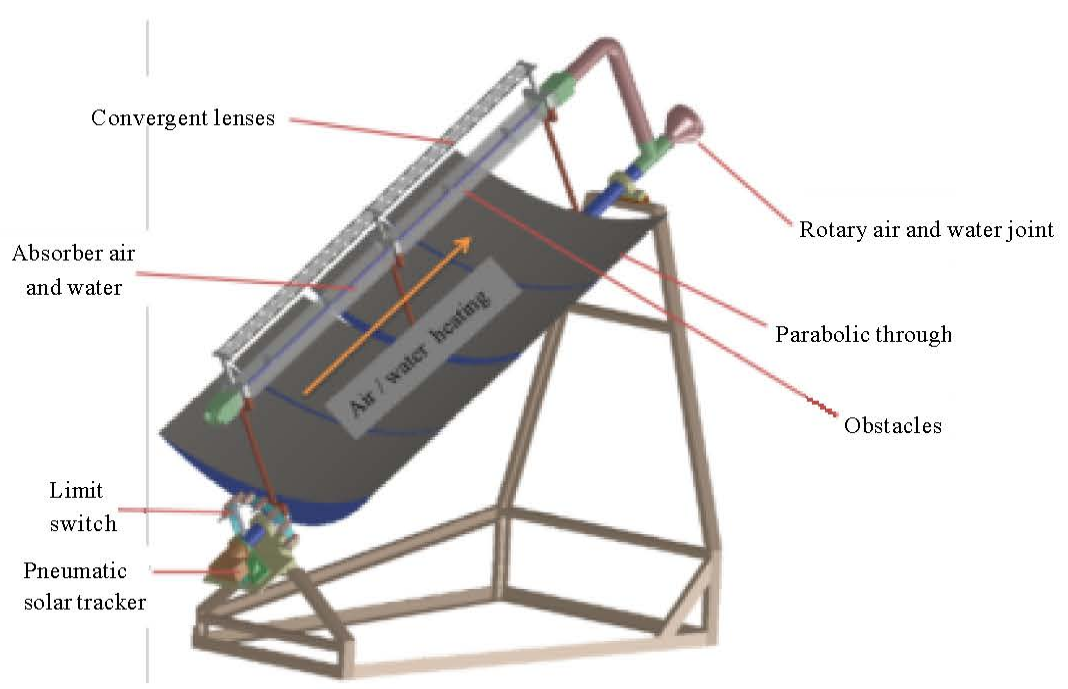

Fig. 2: Schematic of two types of concentrators combined

(a)

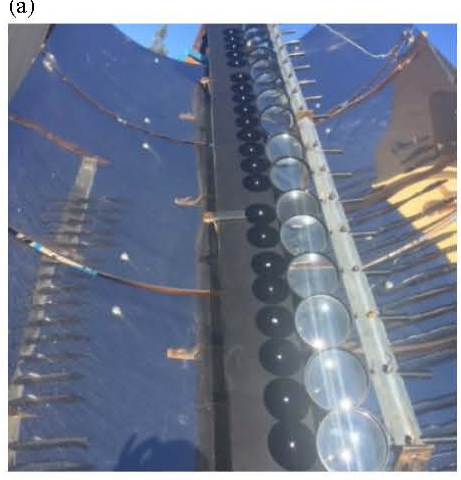

(b)

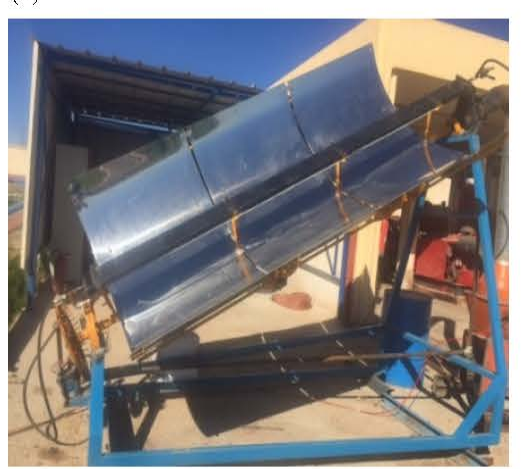

Fig. 3: a, b) The parabolic trough concentrator combined with converging lenses realized

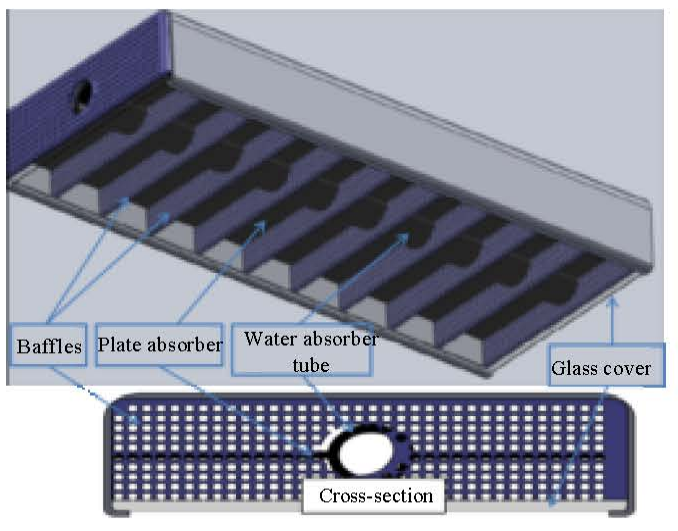

Fig. 4: Receiver having a cover glass on one side only

of the system at Oujda in accordance with the findings of the researches by Bennamoun and Belhamri (2007). When

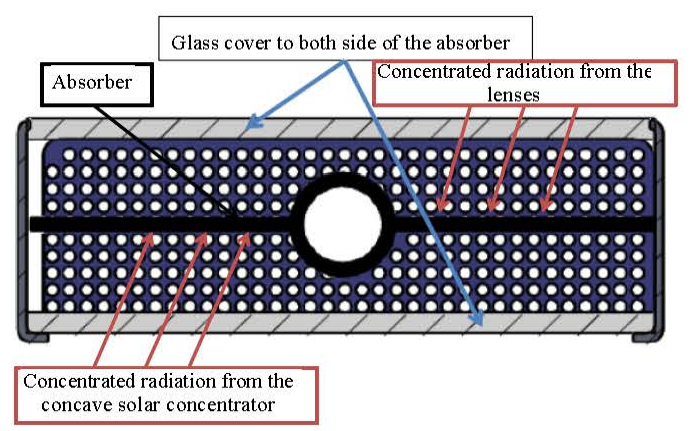

Fig. 5: Receiver having a glass covers on both sides

solar rays hit a surface at an oblique angle, the rays are more spread out. The system was oriented to face the east to maximize the incident solar radiation on the collector. The automatic operation of the dryer with the beginning 
(a)

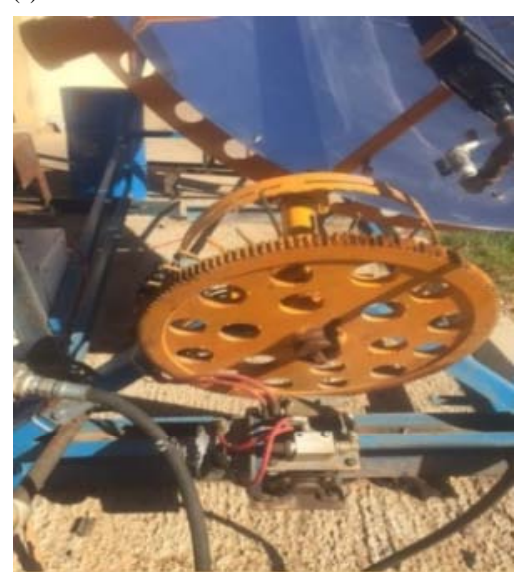

(b)

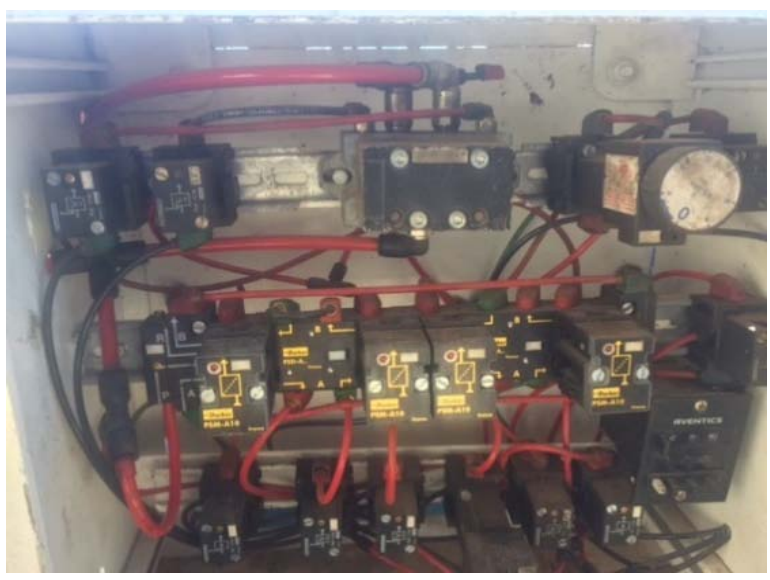

Fig. 6: a, b) Solar tracker developed for explosive atmosphere zone

of the painting cycle has led to the reduction of heat loss and improving the dryer thermal performance. A detailed numerical and experimental analysis of such an optical design system with $3 \mathrm{~m}$ focal length and $1.8 \mathrm{~m}$ effective aperture was performed in a previous study.

Air heating unit: The collector is orientated in a North-South direction and tracking the Sun from East to West. The stainless reflector surface of $6 \mathrm{~m}^{2}$ focuses the Sun's radiation on a receiver canal, called a solar absorber, along the focal line of reflector length of $3 \mathrm{~m}$. A series of 27 convergent lenses focus the radiation on the opposite part of the same receiver.

The absorber channel, having a rectangular shape was made in galvanized sheet on which was fixed the water tube and was inserted crosswise and covered with a layer of black paint. The bottom of the absorbing channel is ordinary glass, allowing passage of the concentrated solar radiation to the focal plane within the channel. Figure 6 shows some improvements made at the flat absorber, creating two areas greenhouse heating for same receiver. This aims to compensate the heat losses at the absorber by the added energy provided by the converging lenses.

The thermal oil has been replaced by air and water as HTF (Heat Transfer Fluid) which are free of cost, nonpolluting and the air has practically no operating temperature limit. The receiver implements a longitudinal flow design where the temperature gain is obtained at the passage of air between obstacles which circulates hot water. The cross-section of the solar air/water receiver is shown schematically in Fig. 5-6. The receiver has a dimension of $150 \times 50 \mathrm{~mm}$ with a face glass cover in Fig. 5 and with the glass cover on both sides of the planar absorber as shown in Fig. 6. Thus, the absorber receives heat energy on one side from the parabolic trough concentrator and on the other side from the converging lenses.

Experimental procedure: Painted cylinders transported by a motorized chain conveyor enter one by one into the drying unit. The fan is operated automatically with a speed adjusted to the optimum air during the drying experiments. The air is preheated the first time during its passage through the obstacles fixed on the planar absorber and the hot water tube and then these two natural fluids (air and water) will be sent to a heat exchanger to raise the temperature of the drying air up to $85^{\circ} \mathrm{C}$. The air charged with solvents is discharged outside of the working environment using the paint booth extractor. Quality paint has been tested manually and with the specific tape.

The hot water then passes through a condenser and circulates in a closed circuit by thermo-siphon-induced flow. The height position of the condenser, relative to the concentrator, ensures free passage of water in the absorber without the need to use a circulation pump rate in the solar receiver. The condenser is equipped with a safety valve, a pressure gauge and a tap for the makeup water.

The solar tracker used is specific to the explosive medium using a pneumatic motor and a pneumatic control. The control circuit is pneumatic and the limit has been provided at the beginning and end of the research day. Timers are adjustable, depending on the season with the opportunity to order a manually rotating hub as shown in Fig. 7.

The timing for drying should be equal or inferior to $40 \mathrm{sec}$ to obtain the desired result. The solvents content in each cylinder is about $0.01 \mathrm{~L}$. The 


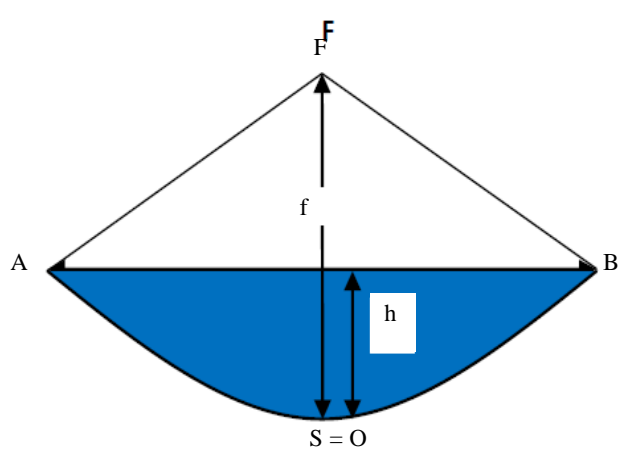

Fig. 7: Parabolic cross section of the collector

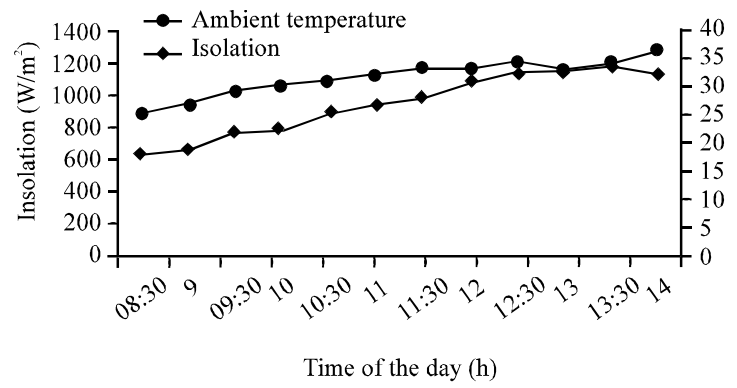

Fig. 8: Variation of the ambient temperature and the insolation during a day in April 2018

measurement of temperatures, solar radiation and air speed is done every $15 \mathrm{~min}$. The drying operation starts at $08: 30$ and stops at 15:30.

System analysis: The dimensions and the thermal energy produced by the parabolic trough concentrator are calculated using the following formulas. Concentration ratio is based on the concept of surface (Guerraiche et al., 2011):

$$
\mathrm{Cg}=\frac{\mathrm{Sc}}{\mathrm{Sr}}
$$

Distance focal line (Fig. 8):

$$
\mathrm{f}=\frac{\mathrm{r}^{2}}{(4 \times \mathrm{h})} \mathrm{r}=\frac{\mathrm{AB}}{2}
$$

The power output is the one recovered by the collector at the absorber level, it can be determined by the following expression:

$$
Q_{u}=Q_{a}-Q_{p}
$$

Thermal losses reduce the energy received in the opening of a cylindrical parabolic concentrator the power consumption is expressed as follows, $Q_{p}$ : Power corresponding to thermal losses:

$$
\mathrm{Q}_{\mathrm{a}}=\mathrm{I}_{\mathrm{c}} \cdot \mathrm{S}_{\mathrm{r}} \cdot \tau \cdot \alpha \cdot \rho
$$

The convective losses $\mathrm{Q}_{\mathrm{p.cv}}$ and the radiation losses $Q_{p . r}$ at the receiver are determined by the expression:

$$
\begin{gathered}
Q_{p . c v}=h_{c y} S_{r} \cdot\left(T_{r}-T_{a}\right) \\
Q_{p . r}=\varepsilon_{r} \cdot \sigma S_{r} \cdot\left(T^{4}-T^{-1 i e l}{ }^{4}\right) \\
Q_{p}=Q_{p . c v}+Q_{p . r}
\end{gathered}
$$

These thermal losses are proportional to the absorber surface and the factors $\left(\mathrm{T}_{\mathrm{r}}-\mathrm{T}_{\mathrm{a}}\right)$ and $\left(\mathrm{Tr}^{4}-\mathrm{Tciel}^{4}\right)$ related to the absorber temperature and the ambient temperature. Our goal is to reduce these two factors by the additional energy provided by the converging lenses and the greenhouse effect created on the second side of the absorber (Fig. 2 and 3). Indeed, the energy produced by the convergent lenses allows the significant increase $\mathrm{T}_{\mathrm{a}}$. For calculating the outlet heat transfer fluid temperature, the following equation was used:

$$
\mathrm{T}_{\mathrm{s}}=\mathrm{T}_{\mathrm{e}}+\frac{\mathrm{Q}_{\mathrm{u}}}{\mathrm{Cp} \cdot \mathrm{M}}
$$

\section{RESULTS AND DISCUSSION}

A solar dryer has been manufactured and tested in a butane gas filling plant (Oujda, Morocco). This dryer will be used for drying gas bottles after washing and after painting in a short time. The bottles pass one by one automatically under the drying unit. The drying air velocity was fixed at $0.9 \mathrm{~m} / \mathrm{sec}$. The dryer performance wastested in 3 days, the dryer was tested in an environment with average relative humidity $30 \%$ and average ambient temperature $25^{\circ} \mathrm{C}$.

Solar radiation and ambient temperature values: The change of solar radiation and ambient temperature on April 102 is shown in Fig. 9. A solar maximum intensity $1200 \mathrm{~W} / \mathrm{m}^{2}$ was observed at an ambient temperature of $35^{\circ} \mathrm{C}$. It increases from the Sun rising to reach a maximum at mid-day before decreasing again until cancelling at night. The use of solar energy is well adapted to the applications for which the needs coincide with the sunniest hours. 


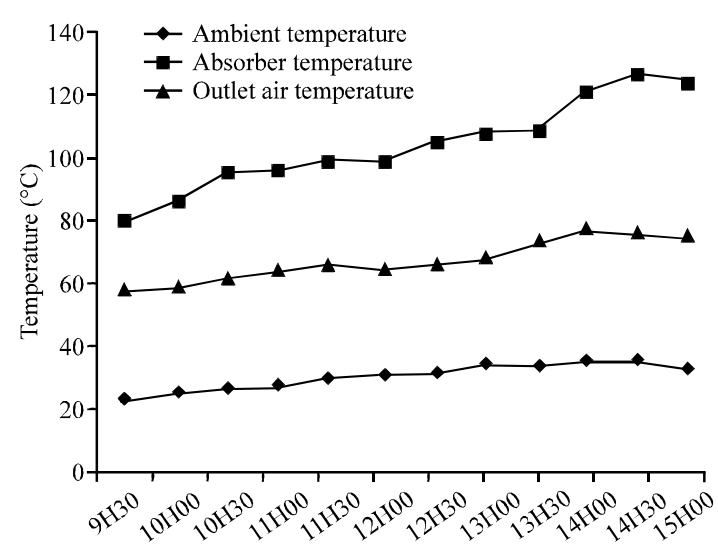

Time of the day (h)

Fig. 9: Drying air temperature

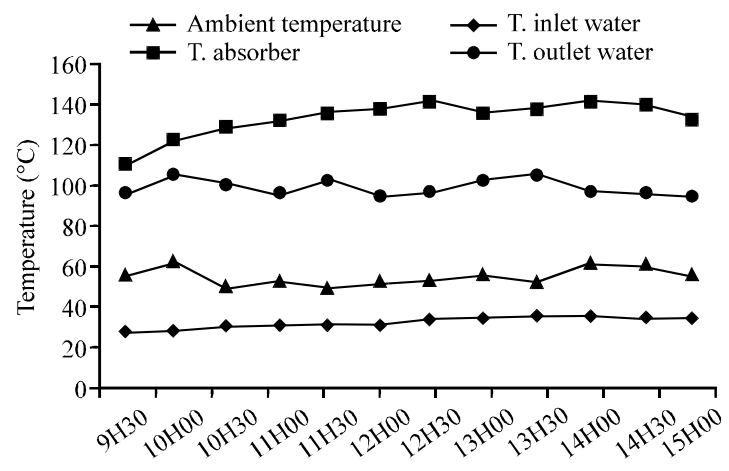

Time (h)

Fig. 10: Inlet-outlet water temperature in absorber

The impact of the simultaneous use of two natural heat transfer fluids: As seen in Fig. 10 and 11 by using exchanger air/water and two heat transfer fluids, the drying air temperature has improved on average by $05^{\circ} \mathrm{C}$. However, the use of obstacles throughout the absorber allowed the improvement of the drying air temperature. The maximum temperature of the drying air recorded during hours of peak sunlight was $85^{\circ} \mathrm{C}$.

The average drying air temperature recorded was $65^{\circ} \mathrm{C}$, the high temperature of the absorber and high convective and radiative heat transfer. The water circulates in a closed circuit and the temperature at the receiver inlet is high in addition to the thermal insulation of the hot water circuit and this is responsible for the rise in the air temperature.

Impact of the combination of convergent lenses with the parabolic concentrator: We find in Fig. 10 and 11, the improvement of the absorber Temperature $\mathrm{T}_{\mathrm{a}}$ by the combination of convergent lenses having only a surface

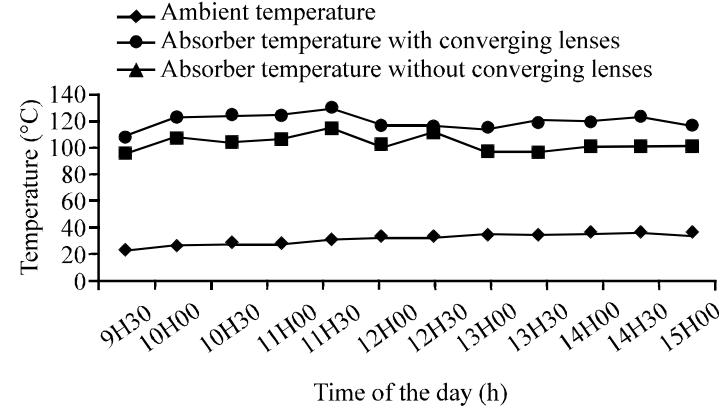

Fig. 11: Lenses impact on the absorber temperature

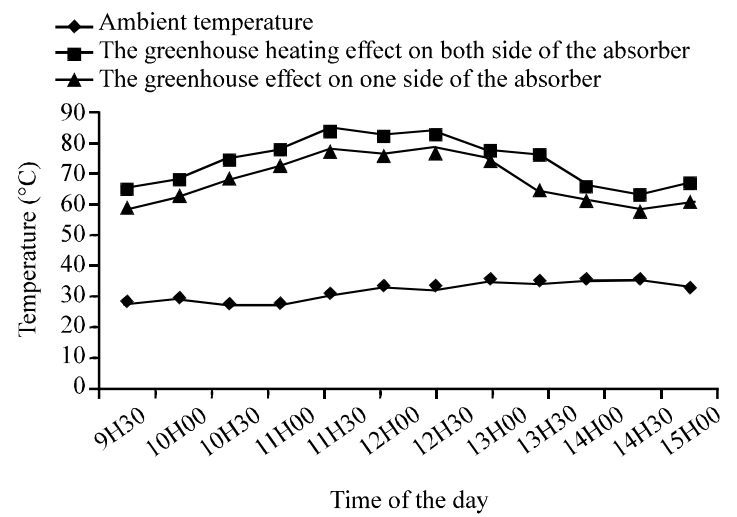

Fig. 12: The impact of the greenhouse effect on both sides of the absorber on the drying air temperature

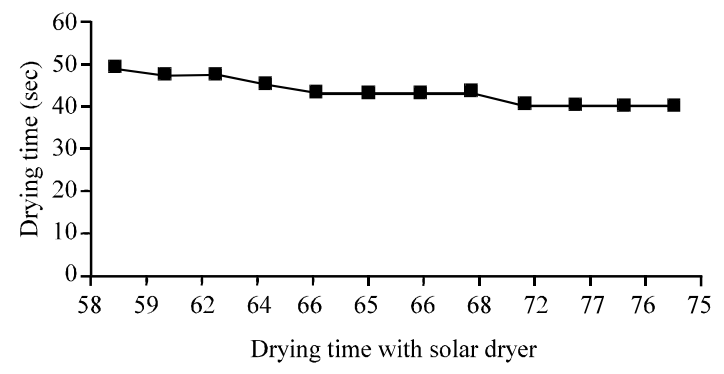

Fig. 13: Drying time with solar dryer

of $0.79 \mathrm{~cm}^{2}$ with the parabolic concentrator. Given these results, it is planned to put Fresnel lenses having a surface area of $40 \mathrm{~cm}^{2}$ to increase the absorber temperature $\mathrm{T}_{\mathrm{a}}$ and thus, reduce heat losses at the receiver. In Fig. 12 we see the impact of the technique of creating the greenhouse effect on both sides of the absorber, the improvement of the drying air temperature is on average of $07^{\circ} \mathrm{C}$.

The impact of drying air temperature: Figure 13 represents the temperature variation according to time in 
the drying unit for an optimum airflow $\left(500 \mathrm{~m}^{3} / \mathrm{h}\right)$. There was a significant reduction in the drying time from $420 \mathrm{sec}$ natural with dryingto about $40 \mathrm{sec}$ using our solar dryerand without the need to store the painting cylinders on the floor. The performance of the painting activity was increased by over $43 \%$.

\section{CONCLUSION}

A forced convection solar dryer using a parabolic concentrator combined with a series of convergent lenses, the whole is tilted at an angle of $34^{\circ}$. This dryerwas designed, fabricated and tested for drying painted gas cylinders. Firstly, our work demonstrates the importance of this new technique which consists in the combination of 2 types of concentrators for the sameabsorber. Secondly, dryer performances were improved by reducing losses due to the greenhouse heating created on both sides of the absorber. An increase in the absorber temperature of more than $15^{\circ} \mathrm{C}$ has been reached. We also deduce the simultaneous use of 2 natural heat exchange fluids (air and water) resulted in the improvement of the drying air temperature and reaches $85^{\circ} \mathrm{C}$. The performance was maintained by sun tracking and tilting the concentrators at a $34^{\circ}$ angle. The Sun is tracked by a pneumatic tracker developed by us and which is specific for an explosive atmosphere.

This dryer reduced drying time from $420 \mathrm{sec}$ to just $40 \mathrm{sec}$ with better paint quality. This dryer allowed the improvement of the productivity of the painting activity of more than $43 \%$. On the health and safety side, employees are not exposed to solvents. In perspective and given the results obtained, we will increase the Fresnel lens surface from $0.79-40 \mathrm{~cm}^{2}$.

\section{ACKNOWLEDGEMENT}

This research work was funded by the company Salam Gas, Morocco in cooperation with Engineering School of Applied Sciences, Mohamed first University, Oujda, Morocco.

\section{REFERENCES}

Bader, R., A. Pedretti and A. Steinfeld, 2011. A 9-m-aperture solar parabolic trough concentrator based on a multilayer polymer mirror membrane mounted on a concrete structure. J. Sol. Energy Eng., 133: $1-12$.

Beach, J.R., J. Raven, C. Ingram, M. Bailey and D. Johns et al., 1997. The effects on asthmatics of exposure to a conventional water-based and a volatile organic compound-free paint. Eur. Respir. J., 10: 563-566.
Benli, H. and A. Durmus, 2009. Performance analysis of a latent heat storage system with phase change material for new designed solar collectors in greenhouse heating. Sol. Energy, 83: 2109-2119.

Bennamoun, L. and A. Belhamri, 2007. Contribution to the feasibility study of solar dryers for agricultural products. Renewable Energies Rev., 1: 201-204.

Blair, R., G. Calota, A. Crossman, F. Drake and K. O'Keefe, 2005. Design of a solar powered fruit and vegetable drier. Master Thesis, Northeastern University, Boston, Massachusetts.

Bulut, H. and F. Durmaz, 2006. Designing, manufacturing and experimental analysis of solar air collector.I. Proceedings of the 2006 International Conference on National Solar and Hydrogen Energy (UGHEK'2006), June 21-23, 2006, Eskisehir, Turkey, pp: 21-23.

Christensen, C., 1998. Federal energy management program. United States Department of Energy, Washington D.C., USA. https://www.energy. gov/eere/femp/about-federal-energy-management-p rogram- 0

Cordeau, S., 2010. The performance of a solar air pre-heater system for the ventilation of two commercial poultry barns. MS Thesis, McGill University, Montreal, Canada.

Forson, F.K., M.A. Nazha and H. Rajakaruna, 2003. Experimental and simulation studies on a single pass, double duct solar air heater. Energy Convers. Manage., 44: 1209-1227.

Fudholi, A., K. Sopian, M.H. Ruslan, M.A. Alghoul and M.Y. Sulaiman, 2010. Review of solar dryers for agricultural and marine products. Renewable Sustainable Energy Rev., 14: 1-30.

Fudholi, A., K. Sopian, M.H. Ruslan, M.Y. Othman and M. Yahya, 2011. Thermal efficiency of double pass solar collector with longitudinal fins absorbers. Am. J. Applied Sci., 8: 254-260.

Gama, A., M. Haddadi and A. Malek, 2008. [Study and realization of a solar parabolic trough concentrator with blind solar tracking (In French)]. Rev. Renewable Energies, 11: 437-451.

Guerraiche, D., A. Benderradji and H. Benmoussa, 2011. [Optical and geometric factors characterizing a cylindro-parabolic concentrator (In French)]. Renewable Energies Rev., 14: 229-238.

Ho, C.D., H.M. Yeh and T.C. Chen, 2011. Collector efficiency of upward-type double pass solar air heaters with fins attached. Int. Commun. Heat Mass Transfer, 38: 49-56.

Honsberg, C., 2008. Solar radiation. University of Delaware, Newark, Delaware. 
Karim, M.A. and M.N.A. Hawlader, 2004. Development of solar air collectors for drying applications. Energy Convers. Manage., 45: 329-344.

Keey, R.B., 1992. Drying of loose and particulate materials. Drying Technol., 10: 1139-1141.

Momin, A.M.E., J.S. Saini and S.C. Solanki, 2002. Heat transfer and friction in solar air heater duct with $\mathrm{V}$-shaped rib roughness on absorber plate. Intl. J. Heat Mass Transfer, 45: 3383-3396.

Pronk, A., L. Preller, M. Raulf-Heimsoth, I.C.L. Jonkers and J.W. Lammers et al., 2007. Respiratory symptoms, sensitization and exposure-response relationships in spray painters exposed to isocyanates. Am. J. Respire. Crit. Care Med., 176: 1090-1097.
Sokhansanj, S. and G.J. Schoenau, 1991. Evaluation of a solar collector system with thermal storage for preheating ventilation air in farm buildings. Energy Convers. Manage., 32: 183-189.

Teyeb, A., L. Dehmani, A. Ben Ezzine, C. Kerkeni and C. Kaabi, 2006. [Study of the performance of a through storageconcentrator (In French)]. Renewable Energies Rev., 9: 135-141.

Turgut, O. and N. Onur, 2000. Design and performance of a special solar collector and its application to drying of agricultural products. J. Inst. Sci. Technol. Gazi Univ., 13: 639-648.

Youcef-Ali, S. and J.Y. Desmons, 2006. Numerical and experimental study of a solar equipped with offset rectangular plate fin absorber plate. Renewable Energy, 31: 2063-2075. 